\title{
Rotating relativistic stars: Matching conditions and kinematical properties
}

\author{
L. M. González-Romero* \\ Departamento de Física Teórica II, Facultad de Ciencias Físicas, Universidad Complutense, 28040-Madrid, Spain
}

(Received 22 October 2002; published 26 March 2003)

\begin{abstract}
In the framework of general relativity, a description of the matching conditions between two rotating perfect fluids spacetimes in terms of the kinematical properties of the fluids is introduced. The Einstein and Darmois equations are written using coordinates adapted to the boundary separating both spacetimes. The functions appearing in the equations have an immediate physical interpretation. The analysis is extended to the case of matching a perfect fluid spacetime (star interior) with a vacuum spacetime (gravitational field outside the star). By solving a boundary problem for a first order partial differential equation ("master equation") we define an exterior tetrad such that the matching conditions and the Einstein equations, for this case, reproduce those of the two-fluid problem. The formalism is applied to a particular static spherically symmetric star and to the Kerr metric.
\end{abstract}

DOI: 10.1103/PhysRevD.67.064011

PACS number(s): 04.40.Dg, 04.20.-q, 95.30.Sf, 97.10.Kc

\section{INTRODUCTION}

One of the main subjects of relativistic astrophysics is the description of the constitution and evolution of relativistic stars (neutron stars, quarks stars, ... ). These are stars with rapid internal motion and/or a high density such that it is necessary to use general relativity to describe them. Usually, these stars rotate (with rigid or differential rotation). Here we will consider stationary and axisymmetric configurations in circular motion, i.e. in permanent rotation. Trying to describe one of these isolated rotating bodies, we immediately realize that we have to cope with the problem of finding an interior solution (the gravitational field inside the star) and an exterior solution, describing the gravity field produced outside the star. These solutions have to match adequately such that we do not have fictitious forces when crossing the star surface. The condition that the star is an isolated body imposes that the exterior solution has to satisfy a condition of asymptotic flatness. It is obvious from the very beginning that we do not know the boundary of the star a priori. That complicates the situation substantially, and mathematically becomes a free-boundary problem.

The matching or junctions conditions between two spacetimes is a fundamental part of the problem. This subject has been considered in classic works by Darmois [1], Lichnerowicz [2], O'Brien and Synge [3], and Israel [4]. The relation between the different approaches has been considered by Bonnor and Vickers [5]. The expression of these conditions in terms of the Newman-Penrose formalism has been presented in [6]. Null matching surfaces have been studied in several works $[7,8]$ and a formalism valid for hypersurfaces of any constant type is presented in [9]. Junction conditions for general hypersurfaces (changing the type from point to point) are considered in [10]. Symmetry-preserving matchings, especially for stationary and axisymmetric spacetimes, have been studied in [11].

There are interesting results for the particular case of rotating bodies. By solving two integral equations, Neugebauer

\footnotetext{
*Email address: mgromero@fis.ucm.es
}

and Meinel [12] have constructed a general relativistic generalization of the classical zero-pressure Maclaurin disk. The density of the dust disk was determined by computing the jump in one of the metric functions. The uniqueness of the exterior gravitational field has been studied in [13]. The possibility of matching the Wahlquist interior solution with an exterior field has been studied by considering a slow rotation approximation in [14]. The matching of a special class of solutions is presented in [15].

A procedure, based in the monodromy matrix, for matching a given stationary axisymmetric perfect fluid solution to a not necessarily asymptotically flat vacuum exterior is described by Ernst and Hauser in [16].

We would like to mention the numerical codes developed to construct models of rotating relativistic stars [17]. The Einstein equations are integrated numerically with the asymptotic conditions at infinity. These models are very interesting because they produce very accurate results for the global properties of rapidly rotating stars. Let us note that usually those numerical codes use coordinates not adapted to the surface of the star and then, if discontinuous physical properties exist in this surface (for instance, the energy density), they suffer from a reduction of the accuracy due to a high-frequency noise at the surface of the star (Gibbs phenomenon) [18]. In order to improve the accuracy in those models, as well as models with phase transition, some surface-fitted coordinates could be used. In this paper we introduce coordinates of this type, which are exactly adapted to the transition surfaces. It is interesting to note that a scheme due to Bonazzola et al. [19], which uses numerically adapted coordinates in a multi-domain spectral code, produces improved results near the surface of the star.

One way to cope with free-boundary problems is to define coordinates such that the matching hypersurface is given by the vanishing of one coordinate in the description of both the interior and the exterior regions. In this paper, we will introduce coordinates of this kind and we will write down the Einstein equations and the matching conditions (Darmois conditions) using them.

The star interior is usually described as a fluid; here we will use perfect fluids in circular motion with a barotropic 
equation of state. To have a better knowledge of the conditions imposed by the matching process on the kinematical properties of the fluid (vorticity, shear, ... ), we consider the problem of matching two perfect fluids across a surface of constant pressure; for instance, two fluids with different rotation regimes or with different equations of state. Then, we can write the matching conditions by using the kinematical properties of both fluids on the matching hypersurface. This situation is interesting by itself, because it can be used as a model for a star with a phase transition (two regions with different equations of state or rotation laws, separated by a well-defined surface). The results can be used as a guide to the problem of matching an interior fluid solution and an exterior vacuum solution, i.e. to obtain an isolated rotating star model. In particular, when matching a fluid interior region with a vacuum exterior region, we will use the gauge freedom, that exists in vacuum spacetimes in defining an orthonormal tetrad, to reproduce the properties of the junction process of two fluids. Also, we choose coordinates such that the matching surface is given by the condition of the vanishing of one of the coordinates. This coordinate is defined in terms of the tetrad in a manner we will describe below.

We will see how this choice of gauge and coordinates has the by-product of a simplified version of the equations in the interior and exterior regions. Also, in the interior region the functions that we find in the equations (Einstein and Darmois equations) have an immediate interpretation in terms of the properties of the fluid. For the exterior region, we will prove that, in general, it is possible to define, in a unique manner, an orthonormal tetrad verifying all the conditions described above. To obtain this tetrad we have to solve a first-order partial differential equation, which we will call the master equation. In our opinion, this tetrad can be considered as a natural prolongation to the exterior of the intrinsic interior tetrad, which is determined by the fluid velocity.

We apply the formalism to a static spherically symmetry configuration where the entire process can be done in an explicit and analytical manner. This configuration can also be used as a guide for further developments. The master equation for the Kerr metric is analyzed, and some particular exact solutions are obtained and analyzed. These particular solutions are extended for the master equation of more general spacetimes.

\section{DIFFERENTIAL FORM APPROACH FOR ROTATING PERFECT FLUIDS}

To study the properties of the stationary and axisymmetric perfect fluids in circular motion, we use a fluid-adapted orthonormal tetrad formalism which was previously introduced [20]. The Einstein equations are formulated as an exterior differential system where the 1-forms used (kinematical 1 -forms) have an immediate interpretation in terms of the kinematical properties of the fluid (acceleration, vorticity, shear,...). To fix the notation, in this section we will present the definitions of these kinematical 1-forms. For completeness, the exterior differential system equivalent to the Einstein equations will be summarized in the Appendix.
We use a Ricci principal tetrad; in our case this implies that we have the fluid velocity $\theta^{0}$ (timelike) as one of the tetrad elements. Given that we assume that our spacetimes are stationary and axisymmetric, with a circular motion, we can choose another element of the tetrad $\theta^{1}$ (spacelike) in the space of the orbits generated by the Killing fields. The only condition for the other two elements of the tetrad $\theta^{2}$ and $\theta^{3}$ is that they have to lie in the two-dimensional space orthogonal to $\theta^{0}$ and $\theta^{1}$, therefore we have a gauge freedom in choosing them. We introduce 1 -forms $a, w$, and $s$ in the $\left\{\theta^{2}, \theta^{3}\right\}$ space, such that the kinematical properties of the fluid can be written in terms of them. The expansion $\Theta$ vanishes, $a$ is the acceleration 1-form, and the shear and vorticity tensors can be written as follows:

$$
\begin{gathered}
\sigma=\theta^{1} \otimes_{s} s \\
\omega_{T}=\theta^{1} \wedge w .
\end{gathered}
$$

The vanishing torsion equations, the first Bianchi identities, the Einstein field equations, and the Euler equation can be written using these kinematical 1-forms and two other 1 -forms $b$ and $\nu$, which are also in the $\left\{\theta^{2}, \theta^{3}\right\}$ space. These $b$ and $\nu 1$-forms can be interpreted as, respectively, the expansion of the volume element in $\left\{\theta^{0}, \theta^{1}\right\}$ space and the connection in the $\left\{\theta^{2}, \theta^{3}\right\}$ space. For an explicit version of the exterior system see the Appendix and [20,21]. The energy density $\mu$ and the pressure $p$ parametrize the thermodynamic properties of the fluid.

Let us mention that the symmetry of our problem impose that all the functions appearing in our formulation depend only on two coordinates whose differentials are in the space generated by $\theta^{2}, \theta^{3}$.

\section{KINEMATICAL PROPERTIES AND SECOND FUNDAMENTAL FORM OF THE MATCHING HYPERSURFACE}

In the problem of matching two spacetimes which are solutions of the Einstein equations, the regularity conditions impose that the first and the second fundamental forms of the matching hypersurface coincide when they are calculated both from the inside or from the outside. If one or both solutions describe perfect fluids, it is a natural question to ask if the matching conditions can be written using the kinematical properties of the fluids. In this manner, we could have a better knowledge of the physical implications of the matching process.

To express the matching conditions in terms of the kinematical properties of the perfect fluid, we have to write down the second fundamental form of the matching hypersurface using them. We can parametrize the matching hypersurface by its normal 1-form; in our case, a stationary and axisymmetric perfect fluid in circular motion, and if the matching surface is described by the equation $p=$ const, the normal 1-form can be written as follows:

$$
n \in \operatorname{Lin}\left\{\theta^{2}, \theta^{3}\right\} ; \quad n \equiv n_{2} \theta^{2}+n_{3} \theta^{3}
$$


where $n_{2}^{2}+n_{3}^{2}=1$. Let us take an extension of $n$ on a neighborhood of the matching hypersurface. The covariant derivative of $n$ can be written as

$$
\begin{aligned}
\nabla n= & \left(\gamma_{020} n_{2}+\gamma_{030} n_{3}\right) \theta^{0} \otimes \theta^{0}+\left(\gamma_{021} n_{2}+\gamma_{031} n_{3}\right) \\
& \times\left(\theta^{0} \otimes \theta^{1}+\theta^{1} \otimes \theta^{0}\right)+\left(\gamma_{121} n_{2}+\gamma_{131} n_{3}\right) \theta^{1} \otimes \theta^{1}+\theta^{2} \\
& \otimes\left(d n_{2}+\Gamma_{23} n_{3}\right)+\theta^{3} \otimes\left(d n_{3}-\Gamma_{23} n_{2}\right)
\end{aligned}
$$

where $\gamma_{a b c}$ are the Ricci rotation coefficients of the Riemannian connection and $\Gamma_{23} \equiv \gamma_{232} \theta^{2}+\gamma_{233} \theta^{3}$ is the induced Riemannian connection in the $\left\{\theta^{2}, \theta^{3}\right\}$ subspace. When $\theta^{0}$ $=u, u$ being the velocity of the fluid, we can rewrite $\nabla n$ as follows:

$$
\begin{aligned}
\nabla n= & -a \cdot n \quad \theta^{0} \otimes \theta^{0}+\frac{1}{2}(s-w) \cdot n\left(\theta^{0} \otimes \theta^{1}+\theta^{1} \otimes \theta^{0}\right) \\
& +(b-a) \cdot n \quad \theta^{1} \otimes \theta^{1}+T \otimes[d \alpha-\nu]
\end{aligned}
$$

where $T \equiv-n_{3} \theta^{2}+n_{2} \theta^{3}, n_{2}=\cos \alpha, n_{3}=\sin \alpha$, and - indicates the scalar product in the $\left\{\theta^{2}, \theta^{3}\right\}$ subspace. The second fundamental form of the hypersurface $p=$ const is obtained by projecting this covariant derivative on the hypersurface (i.e. calculating the pull-back of this covariant derivative on it). The result is independent of the extension used for $n$ [22].

\section{THE TWO-FLUID PROBLEM}

Let us consider the case when the two spacetimes that we would like to match describe two configurations of rotating perfect fluid (for instance, imagine that we have an inner core of a rotating star in rigid rotation, and the outer part of the star in a differentially rotating regime, or two regions with different equations of state separated by a well-defined surface). We impose that the matching hypersurface $\Sigma$ be a constant-pressure hypersurface $\left(p=p_{0}\right)$; the velocity of the fluid $u=\theta^{0}$ and $\theta^{1}$ are then tangents to $\Sigma$.

For both fluids, from the Euler equation we have that $d p=-(\mu+p) a$, and $n= \pm a /|a|$. We also assume that the fluids have a barotropic equation of state $\mu=\mu(p)$; then we can write $a=d U$, and therefore the matching surface can be described by the equation $U=$ const. By using the freedom in the definition of $U$, we can impose that the matching hypersurface $\Sigma$ is described, in both spacetimes, by the equation $U=0$. If $b \wedge a \neq 0$ (the other case, which includes cylindrical symmetry and dust fluids, is a degenerate one and can be considered in a separated manner [21]) we can use as our coordinates in the $\left\{\theta^{2}, \theta^{3}\right\}$ space $U$ and other coordinate $v$ defined up to a constant by the equation $b=d v$ (the constant can be chosen such that $v_{\text {in } \mid \Sigma}=v_{o u t \mid \Sigma}$ ). Note that for stationary and axisymmetric spacetimes, with no more symmetries, this coordinate $v$ is invariantly defined (up to a constant) because we have

$$
d\left(\theta^{0} \wedge \theta^{1}\right)=b \wedge\left(\theta^{0} \wedge \theta^{1}\right)
$$

where $\theta^{0} \wedge \theta^{1}$ is the volume element in the space of the orbits of the Killing fields and $d b=0$ (the Appendix). Therefore we can write $b=d v$.
Then the kinematical 1-forms can be written as follows (see the Appendix):

$$
\begin{aligned}
& a=d U \\
& b=d v \\
& w=\hat{\kappa} e^{\xi}\left(\varpi_{v} d v+\varpi_{U} d U\right) \\
& s=\hat{\epsilon} e^{-\xi}\left(\varpi_{v} d v+\varpi_{U} d U\right) \\
& \nu=\nu_{v} d v+\nu_{U} d U .
\end{aligned}
$$

Now, we align one of the components of our orthonormal tetrad with the invariant 1 -form $b$, so that $\theta^{2}=G d v$. If we parametrize the duality operator in $\left\{\theta^{2}, \theta^{3}\right\}$ subspace by $\star d v=\left(1 / \sqrt{N-f^{2}}\right)(f d v+d U)\left(N>f^{2}\right), \quad$ and choose $\theta^{3}$ $=\star \theta^{2}$, then we have

$$
\theta^{3}=\frac{G}{\sqrt{N-f^{2}}}(f d v+d U)
$$

where $G, f$ and $N$ are functions of $U$ and $v$ (a similar parametrization, especially adapted for interior regions, has been used in [21]. Here, we have modified it to be also useful for vacuum spacetimes). Therefore, we can write the spacetime metric as

$$
\begin{aligned}
d s^{2}= & -\theta^{0} \otimes \theta^{0}+\theta^{1} \otimes \theta^{1}+\frac{G}{N-f^{2}}\left(N d v^{2}+d U^{2}+2 f d U d v\right) \\
= & -\theta^{0} \otimes \theta^{0}+\theta^{1} \otimes \theta^{1} \\
& +\frac{|a|^{2} d v^{2}+|b|^{2} d U^{2}-2(b \cdot a) d U d v}{|a|^{2}|b|^{2}-(b \cdot a)^{2}}
\end{aligned}
$$

Note that $G, f$ and $N$ have an immediate interpretation in terms of the kinematical 1-forms $a$ and $b$

$$
\begin{aligned}
& G=\frac{1}{|b|^{2}} \\
& N=\frac{|a|^{2}}{|b|^{2}} \\
& f=-\frac{b \cdot a}{|b|^{2}}=-\frac{|a|}{|b|} \cos (b, a) .
\end{aligned}
$$

The Einstein equations can be written as follows: 


$$
\begin{aligned}
& N_{v}=-4 p G N+\left(2-2 f_{U}-m n f \varpi_{U}^{2}\right) N \\
& +\left[8 f^{2} p+(\mu+3 p) f\right] G+4 f f_{v}-2 f^{2} \\
& -m n f\left(\varpi_{v}^{2}-2 \varpi_{U} \varpi_{v} f\right) \\
& N_{U}=-m n \varpi_{U}^{2} N+[4 f p+(\mu+3 p)] G+2 f_{v} \\
& -m n\left(\varpi_{v}^{2}-2 \varpi_{U} \varpi_{v} f\right) \\
& G_{U}=\left[-2-n^{2} \varpi_{U} \varpi_{v}-\left(4-n^{2} \varpi_{U}^{2}\right) f\right] G \\
& G_{v}=-2 p G^{2}+\left[2-\frac{1}{2} n^{2} \varpi_{v}^{2}-\left(4-n^{2} \varpi_{U}^{2}\right) \frac{N}{2}\right] G \\
& p_{U}=-(\mu+p) \\
& 0=\varpi_{v v}+N \varpi_{u u}-2 f \varpi_{u v}-\varpi_{v}[-2 p G+\lambda(1+2 f \\
& \left.\left.+\xi_{U} f-\xi_{v}\right)\right]-\varpi_{U}\left[-\frac{1}{2} N_{U}+f_{v}+2 p G f\right. \\
& \left.-\lambda\left(f+2 N+\xi_{U} N-\xi_{v} f\right)\right]
\end{aligned}
$$

where $m=\kappa e^{\xi}+\epsilon e^{-\xi}, n=\kappa e^{\xi}-\epsilon e^{-\xi}, \lambda=m / n$ and $\xi=2 U$ $-v+2 h(\varpi)$.

It is interesting to note that Eq. (19) is the integrability condition for Eqs. (16) and (17) for $G_{U}$ and $G_{v}$. Therefore, if we obtain $f$ and $N$ from these equations and substitute the result in the rest of the equations, Eq. (19) is identically satisfied. Given an equation of state $\mu=\mu(p)$, the Euler equation (18) can be solved in order to obtain $p=p(U)$ and $\mu=\mu(U)$. The rotation regime has to be fixed by giving a particular function $h(\varpi)$. Finally, we have two second-order partial differential equations for $G$ and $\varpi$ from Eqs. (14) and (15). Therefore, the Einstein equations can be reduced to this system of two second-order partial differential equations for $G$ and $\varpi$. Once a solution is known for $G$ and $\varpi$ also $f$ and $N$ are known, and the complete metric can be written (see the Appendix).
Let us concentrate in the matching conditions in these coordinates. The first fundamental form on the hypersurface $\Sigma$ can be written as follows:

$$
\begin{aligned}
d s_{\mid \Sigma}^{2} & =-\theta_{\mid \Sigma}^{0} \otimes \theta_{\mid \Sigma}^{0}+\theta_{\mid \Sigma}^{1} \otimes \theta_{\mid \Sigma}^{1}+\left(\frac{|a|^{2}}{|a|^{2}|b|^{2}-(b \cdot a)^{2}}\right)_{\mid \Sigma} d v^{2} \\
& =-\theta_{\mid \Sigma}^{0} \otimes \theta_{\mid \Sigma}^{0}+\theta_{\mid \Sigma}^{1} \otimes \theta_{\mid \Sigma}^{1}+\left(\frac{G N}{N-f^{2}}\right)_{\mid \Sigma} d v^{2}
\end{aligned}
$$

and the second fundamental form

$$
\begin{aligned}
& K= \pm\left\{-|a|_{\mid \Sigma} \theta_{\mid \Sigma}^{0} \otimes \theta_{\mid \Sigma}^{0}+\frac{1}{2}\left[(s-w) \cdot \frac{a}{|a|}\right]_{\mid \Sigma}\right. \\
& \times\left(\theta_{\mid \Sigma}^{0} \otimes \theta_{\mid \Sigma}^{1}+\theta_{\mid \Sigma}^{1} \otimes \theta_{\mid \Sigma}^{0}\right)+\left[(b-a) \cdot \frac{a}{|a|}\right]_{\mid \Sigma} \theta_{\mid \Sigma}^{1} \otimes \theta_{\mid \Sigma}^{1} \\
& \left.+\left[T_{v}(d \alpha-\nu)_{v}\right]_{\mid \Sigma} d v^{2}\right\} \\
& = \pm\left\{-\sqrt{\frac{N}{G}} \theta_{\mid \Sigma}^{0} \otimes \theta_{\mid \Sigma}^{0}+\frac{1}{\sqrt{N G}}\left(\hat{\epsilon} e^{-\xi}-\hat{\kappa} e^{\xi}\right)\right. \\
& \times\left(N \varpi_{U}-f \varpi_{v}\right) \theta_{\mid \Sigma}^{0} \otimes_{S} \theta_{\mid \Sigma}^{1}+\left(-\frac{f}{\sqrt{N G}}-\sqrt{\frac{N}{G}}\right) \theta_{\mid \Sigma}^{1} \\
& \otimes \theta_{\mid \Sigma}^{1}-\frac{\sqrt{N G}}{N-f^{2}}\left[\sqrt{N}\left(\frac{f}{\sqrt{N}}\right)_{v}-f \frac{G_{v}}{2 G}+N \frac{G_{U}}{2 G}\right. \\
& \left.-f(1-2 p G)] d v^{2}\right\}
\end{aligned}
$$

where all the function are evaluated in $U=0$. To obtain the last expression for $K$ the Einstein equations have been used.

We impose that there does not exist any discontinuity in the fluid velocity on $\Sigma$, i.e. $\theta_{\left.i n\right|_{\Sigma}}^{0}=\theta_{\left.o u t\right|_{\Sigma}}^{0}$ this condition implies that

$$
\left\{\begin{array}{lll}
s_{i n_{\mid \Sigma}} & = & s_{\text {out }_{\mid \Sigma}} \\
w_{\text {in }} & = & w_{\text {out }} \\
b_{\text {in }} & = & b_{\text {out }}
\end{array}\right\} \Leftrightarrow\left\{\begin{array}{ccc}
(h(\varpi))_{\text {in }} & = & (h(\varpi))_{\text {out }} \\
\left(\varpi_{v}\right)_{\text {in }} & = & \left(\varpi_{v}\right)_{\text {out }} \\
v_{\text {in }} & & \\
\end{array}\right\} .
$$

Therefore, we can define a unique velocity of the fluid on $\Sigma$ as $\theta_{\Sigma}^{0} \equiv \theta_{\text {in }\left.\right|_{\Sigma}}^{0}=\theta_{\text {out }\left.\right|_{\Sigma}}^{0}$, associated with $\theta_{\Sigma}^{0}$ we have its kinematical properties and 1-forms $w_{\Sigma}, s_{\Sigma}, b_{\Sigma}$ on the surface. It is easy to check that these 1 -forms can be obtained by projecting the 1 -forms defined for the interior and exterior fluids on the hypersurface $\Sigma$ (the pull-back commutes with the exterior derivative and the exterior product). Then we have 


$$
\begin{aligned}
& a_{\Sigma}=0 \\
& b_{\Sigma}=d v \\
& w_{\Sigma}=\hat{\kappa} e^{-v+2 h(\varpi)}\left(\varpi_{v}\right) d v
\end{aligned}
$$

$$
s_{\Sigma}=\hat{\epsilon} e^{+v-2 h(\varpi)}\left(\varpi_{v}\right) d v
$$

The continuity of the first and second fundamental forms (Darmois conditions) is equivalent to

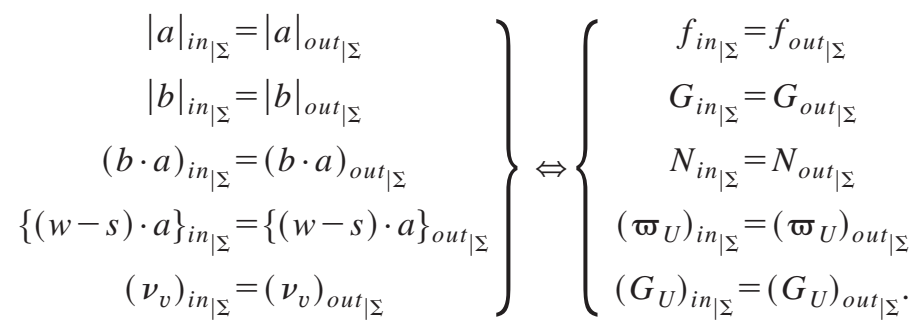

From the equations in Eq. (22), the continuity of the component $d v^{2}$ of the first fundamental form, and the components $\theta_{\mid \Sigma}^{0} \otimes \theta_{\mid \Sigma}^{0}, \theta_{\mid \Sigma}^{0} \otimes \theta_{\mid \Sigma}^{1}$, and $\theta_{\mid \Sigma}^{1} \otimes \theta_{\mid \Sigma}^{1}$ of the second fundamental form we obtain the continuity of $f, G, N$, and $\varpi_{U}$. Using the Einstein equations [in particular, Eq. (17)] we have that $p_{\text {in }_{\mid \Sigma}}=p_{\text {out }_{\mid \Sigma}}$. From the $d v^{2}$ component of the second fundamental form the continuity of $G_{U}$ in $\Sigma$ is derived.

It easy to check, using the previous relations, that $|w|_{\text {in }_{\mid \Sigma}}=|w|_{\text {out }_{\mid \Sigma}}, \quad|s|_{\text {in }_{\mid \Sigma}}=|s|_{\text {out }_{\mid \Sigma}}, \quad(b \cdot w)_{\text {in }_{\mid \Sigma}}=(b \cdot w)_{\text {out }_{\mid \Sigma}}$, and $(a \cdot w)_{i n_{\mid \Sigma}}=(a \cdot w)_{o u t}$. . Therefore, $|a|,|b|,|w|,|s|$, $\cos (a, b), \cos (a, w)$, and $\cos (b, w)$ (the moduli of all the kinematical 1-forms and the angles among them) have to be continuous in $\Sigma$.

There are functions that can be discontinuous on the matching surface. These are, for instance, $f_{U}, N_{U}, p_{U}$, and $\varpi_{u u}$. The discontinuity of these functions can be obtained from the Einstein equations. For these discontinuities we have

$$
\begin{gathered}
{\left[N_{U}\right]=G[\mu]} \\
{\left[f_{U}\right]=\frac{G}{2 N}[\mu]} \\
{\left[p_{U}\right]=-[\mu]} \\
{\left[\varpi_{U U}\right]=\frac{G}{2 N} \varpi_{U}[\mu]}
\end{gathered}
$$

where []$=()_{\text {in }}-()_{\text {out }}$ (we have used the fact that $\dot{h}$ is continuous in $U=0$ ). It is interesting to note that the discontinuities of these functions depend on the discontinuity of $\mu$ (the energy density). These discontinuities can be written in a more intrinsic manner in terms of the derivatives of kinematical properties in the $U$ direction

$$
\left[|a|_{U}\right]=\frac{|b|^{2}}{2|a|}\left[N_{U}\right]=\frac{1}{2|a|}[\mu]
$$

$$
\begin{aligned}
{\left[\cos (b, a)_{U}\right]=} & -\frac{1}{|a|} \cos (b, a)\left[|a|_{U}\right]-\frac{|b|}{|a|}\left[f_{U}\right] \\
= & -\frac{1}{2|a|^{2}}\left\{\cos (b, a)+\frac{|b|}{|a|}\right\}[\mu] \\
2|w|\left[w_{U}\right]= & \frac{\kappa^{2} e^{2 \xi}}{G}\left\{-2 \varpi_{U} \varpi_{v}\left[f_{U}\right]+2\left(N \varpi_{U}-f \varpi_{v}\right)\right. \\
& \left.\times\left[\varpi_{U U}\right]+\varpi_{U}^{2}\left[N_{U}\right]\right\} \\
= & -\kappa^{2} e^{2 \xi} \varpi_{U} \varpi_{v} \frac{|b|^{2}}{|a|^{2}}\left\{1+\frac{|a|}{|b|} \cos (b, a)\right\}[\mu] \\
= & \frac{|w|^{2}}{|a||b| \sin ^{4}(b, a)}\{\cos (b, a)(\cos (b, w) \\
& +\cos (a, w))^{2}-(1+\cos (b, a))^{2} \\
& \times \cos (a, w) \cos (b, w)\} \\
& \times\left\{\frac{|a|}{|b|} \cos (b, a)\right\}[\mu] .
\end{aligned}
$$

As a summary, we can say that the matching conditions in these coordinates impose that

$$
f, G, N, \varpi_{v}, \varpi_{U}, h(\varpi), \text { and } G_{U}
$$

are continuous on $U=0$ (as well as all the $v$ derivatives up to the order of continuous differentiability imposed for a regular point. It is assumed that these derivatives have limits from inside and outside). This is equivalent to the continuity of the moduli of all the kinematical 1-forms and the angles among them.

The two-fluid problem has been reduced to two nonlinear second order partial differential equations with independent variables $U$ and $v$ for $G$ and $\varpi$ in the interior and exterior 
regions. The boundary conditions impose that $G$ and $\varpi$ be continuous and have continuous partial $U$ derivatives on $U$ $=0$.

\section{THE ISOLATED STAR PROBLEM: VACUUM EXTERIOR AND INTERIOR FLUID}

Now, we have to match an interior perfect fluid spacetime (modeling the interior of a star) and an exterior vacuum spacetime (modeling the field produced by the star outside). Following the two-fluid model, we choose a fluid-adapted tetrad for the interior region. In the exterior region, even when we impose that $\theta^{0}$ and $\theta^{1}$ be in the space of the orbits of the two Killing fields, there is a gauge freedom in choosing them. If we take a starting tetrad with caret we can make the following gauge hyperbolic rotation:

$$
\begin{aligned}
& \theta^{0}=\cosh (\lambda) \widehat{\theta^{0}}+\sinh (\lambda) \widehat{\theta^{1}} \\
& \theta^{1}=\sinh (\lambda) \widehat{\theta^{0}}+\cosh (\lambda) \widehat{\theta^{1}}
\end{aligned}
$$

where $\lambda$ is an arbitrary function of the coordinates. We consider a $\lambda$ maintaining the symmetry (i.e. $\lambda$ does not depend on the time and axial coordinates). If we choose some $x$ and $v$ coordinates in the space orthogonal to the orbits of the Killing fields, then, $\lambda$ is a function of $x$ and $v$. In this section, the leading idea is to use this gauge freedom (choose $\lambda$ ) to reproduce the previously developed two-fluid matching process. The main ingredient of the two fluids matching model is a $\theta^{0}$ such that the corresponding acceleration 1-form $a$ verifies that $a=d U$ and the matching hypersurface $\Sigma$ is described by the equation $U=0$. Also, it is satisfied that $\theta_{i n_{\mid \Sigma}}^{0}$ $=\theta_{\text {out }_{\mid \Sigma}}^{0}$.

In the case we are considering now, in the exterior region we have no Euler equation and no baryotropic equation of state implying that $d a=0$, but we have the gauge freedom choosing $\theta^{0}$. Hence, we look for a $\theta^{0}$ that verifies $w \wedge s$ $=0$ and therefore, by the second Bianchi identities, $d a=0$ holds ( $a=d U$, at least locally).

Under the previously considered gauge rotation, the 1 -forms $a, b, w$, and $s$ transform adequately. It is interesting to define auxiliary 1 -forms

$$
\begin{aligned}
& \beta \equiv b-2 a \\
& \delta \equiv w-s \\
& \kappa \equiv w+s
\end{aligned}
$$

such that the transformation relations can be written in the following simple manner:

$$
\begin{aligned}
& b=\hat{b} \\
& \kappa=\hat{\kappa}+2 d \lambda
\end{aligned}
$$

$$
\begin{aligned}
& \beta=\cosh (2 \lambda) \hat{\beta}+\sinh (2 \lambda) \hat{\delta} \\
& \delta=\sinh (2 \lambda) \hat{\beta}+\cosh (2 \lambda) \hat{\delta}
\end{aligned}
$$

The condition $w \wedge s=0$ is equivalent to $\kappa \wedge \delta=0$, which in terms of the variables with carets reads

$$
(\hat{\kappa}+2 d \lambda) \wedge[\sinh (2 \lambda) \hat{\beta}+\cosh (2 \lambda) \hat{\delta}]=0 .
$$

We call this equation the differential form master equation. The solutions of this equation can be separated in two cases: $\delta=0$ and $\delta \neq 0$. The first one, when $\delta \equiv \sinh (2 \lambda) \hat{\beta}$ $+\cosh (2 \lambda) \hat{\delta}=0$, implies, by using the field equations, that the spacetime is static. We will not consider this case in detail here, as it can be treated in a separate and simpler manner.

In the second case, we have $\kappa=\alpha \delta$, which in terms of the variables with carets reads as follows:

$$
\hat{\kappa}+2 d \lambda=\alpha[\sinh (2 \lambda) \hat{\beta}+\cosh (2 \lambda) \hat{\delta}]
$$

where $\alpha$ is an arbitrary function of the coordinates $x$ and $v$. This is an equation where we have to determine $\alpha$ and $\lambda$. Note that we can interpret this equation as determining all the possible gauge transformations that from a given starting tetrad with caret give as result a new tetrad verifying $w \wedge s$ $=0 \Leftrightarrow d a=0$. If we start with a tetrad already satisfying $w$ $\wedge_{S}=0$, then the equation determines the transformations that maintain this property. Using coordinates $x$ and $v$ the differential form master equation implies

$$
\begin{aligned}
& 2 \lambda_{, x}=-\hat{\kappa}_{x}+\alpha\left[\sinh (2 \lambda) \hat{\beta}_{x}+\cosh (2 \lambda) \hat{\delta}_{x}\right] \\
& 2 \lambda_{, v}=-\hat{\kappa}_{v}+\alpha\left[\sinh (2 \lambda) \hat{\beta}_{v}+\cosh (2 \lambda) \hat{\delta}_{v}\right] .
\end{aligned}
$$

We will assume that $\sinh (2 \lambda) \hat{\beta}_{x}+\cosh (2 \lambda) \hat{\delta}_{x}$ and $\sinh (2 \lambda) \hat{\beta}_{v}+\cosh (2 \lambda) \hat{\delta}_{v}$ do not vanish, because if both vanish then $\delta=0$ and the solution is static, if only one of them vanishes, for instance the second one (the other case has a similar treatment), we will have one of the following possibilities.

(1) $\hat{\beta}_{v}=\hat{\delta}_{v}=0$ and then the solution of the equation is

$$
\lambda=-\frac{1}{2} \int \hat{k}_{v} d v+F(x)
$$

and

$$
\alpha=\frac{2 \lambda_{, x}+\hat{\kappa}_{x}}{\sinh (2 \lambda) \hat{\beta}_{x}+\cosh (2 \lambda) \hat{\delta}_{x}}
$$


(2) $\hat{\beta}_{v} \neq 0$ and then

$$
\lambda=\frac{1}{2} \operatorname{arctanh}\left(-\frac{\hat{\delta}_{v}}{\hat{\beta}_{v}}\right)
$$

which imposes the following constraint:

$$
\hat{k}_{v}=\frac{1}{1-\left(\frac{\hat{\delta}_{v}}{\hat{\beta}_{v}}\right)^{2}}\left(-\frac{\hat{\delta}_{v}}{\hat{\beta}_{v}}\right)_{v}
$$

and

$$
\alpha=\frac{2 \lambda_{, x}+\hat{\kappa}_{x}}{\sinh (2 \lambda) \hat{\beta}_{x}+\cosh (2 \lambda) \hat{\delta}_{x}} .
$$

Hence we can check, a priori, if we are in one of these cases where the solution to the master equation is known.

In the general case we can obtain $\alpha$ from one of the equations, for instance from the first one, and then substitute the result in the other equation to have

$$
\alpha=\frac{2 \lambda_{, x}+\hat{\kappa}_{x}}{\sinh (2 \lambda) \hat{\beta}_{x}+\cosh (2 \lambda) \hat{\delta}_{x}}
$$

and

$$
\frac{2 \lambda_{, x}+\hat{\kappa}_{x}}{\sinh (2 \lambda) \hat{\beta}_{x}+\cosh (2 \lambda) \hat{\delta}_{x}}=\frac{2 \lambda_{, v}+\hat{\kappa}_{v}}{\sinh (2 \lambda) \hat{\beta}_{v}+\cosh (2 \lambda) \hat{\delta}_{v}} .
$$

This last equation is a first-order quasilinear partial differential equation that can be used to determine $\lambda$. We call this equation the partial differential equation (PDE) master equation; to this equation we have to add the boundary condition $\theta_{\text {in } \mid \Sigma}^{0}=\theta_{\text {out } \mid \Sigma}^{0}$. This fixes $\lambda$ in the surface $\Sigma$ defined by an equation $F(x, v)=0$ [i.e. $\lambda_{\mid \Sigma}=g(v)$ ], but then we have a boundary problem for a quasilinear differential equation, and it is a well known fact that this problem has a unique solution, at least locally around $\Sigma$, unless the boundary condition is a characteristic condition.

Note that the PDE master equation depends on the starting tetrad with caret. To fix the notation we can choose the tetrad with caret as follows:

$$
\begin{aligned}
& \hat{\theta}^{0}=\sqrt{-g_{t t}}\left(d t+\frac{g_{t \varphi}}{g_{t t}} d \varphi\right) \\
& \hat{\theta}^{1}=\sqrt{\frac{g_{t \varphi}^{2}-g_{\varphi \varphi} g_{t t}}{-g_{t t}}} d \varphi
\end{aligned}
$$

where $t$ and $\varphi$ are the coordinates of a non-rotating observer at infinity. For this tetrad we have $s=0$, i.e. it is a rigidly rotating tetrad.

Hence, in a non-characteristic case there is an unique $\theta^{0}$ which satisfied that $w \wedge s=0$ and such that in a given surface $\Sigma$ coincides with a prescribed one. Therefore, the analysis done for the two-fluid problem can be reproduced now with a "virtual" fluid velocity given by this $\theta^{0}$. Then, we obtain exactly the same results as in the two-fluid problem, and the Einstein and Darmois equations can be reduced to a boundary problem for a system of two second-order partial differential equations for two functions in two variables, except that, in this case, we should impose $p_{\text {out }}=0$ and $\mu_{\text {out }}=0$. The conditions imposed by the matching requirements, in the kinematical 1-forms are also the same as in the two-fluid problem.

\section{A. The characteristic case}

Let us analyze the case when the matching hypersurface is a characteristic surface for the PDE master equation. This characteristic surface is given by the condition $\delta_{\text {out } \mid S}=0$ (note that this condition does not imply in general that $\delta$ $=0$ in the exterior region). But if $\delta_{\text {out } \mid \Sigma}=0$, then $\kappa_{\text {out }} \mid \Sigma$ $=0$ because $\kappa_{i n} \propto \delta_{\text {in }}$ and then $\kappa_{\mid \Sigma} \propto \delta_{\mid \Sigma}$ (note that as we have $\theta_{\text {in } \mid \Sigma}^{0}=\theta_{\text {out } \mid \Sigma}^{0}$ then $b_{\text {in } \mid \Sigma}=b_{\text {out } \mid \Sigma}, w_{\text {in } \mid \Sigma}=w_{\text {out } \mid \Sigma}$, and $s_{\text {in } \mid \Sigma}$ $=s_{\text {out } \mid \Sigma}$ and we can refer, without any ambiguity, to the kinematical 1-forms of $\theta_{\mid \Sigma}^{0}$ on the matching hypersurface and write $b_{\mid \Sigma}, w_{\mid \Sigma}$, and $s_{\mid \Sigma}$, equivalently $\delta_{\mid \Sigma}$ and $\kappa_{\mid \Sigma}$ ). From the vanishing torsion equations, we have that we can write the first fundamental form of the surface as follows:

$$
d s_{\mid \Sigma}^{2}=-d t^{2}+e^{2 v} d \varphi^{2}+h(v) d v^{2}
$$

where $u_{\mid \Sigma}= \pm \partial / \partial t\left(\theta_{\mid \Sigma}^{0}=\mp d t\right)$. Using an extension of these coordinates to the exterior region the metric can be written as

$$
\begin{aligned}
d s_{\text {out }}^{2}= & g_{t t} d t^{2}+2 g_{t \varphi} d t d \varphi+g_{\varphi \varphi} d \varphi^{2}+g_{v v} d v^{2}+2 g_{x v} d x d v \\
& +g_{x x} d x^{2},
\end{aligned}
$$

and the matching surface will be given by an equation $F(x, v)=0$, such that

$$
\begin{gathered}
g_{t t \mid \Sigma}=-1 \\
g_{t \varphi \mid \Sigma}=0 \\
g_{\varphi \varphi \mid \Sigma}=e^{2 v}
\end{gathered}
$$

and choosing $\theta^{0}=\sqrt{-g_{t t}}\left(d t+\left(g_{t \varphi} / g_{t t}\right) d \varphi\right)$ and $\theta^{1}$ $=\sqrt{\left(g_{\varphi \varphi} g_{t t}-g_{t \varphi}^{2}\right) / g_{t t}} d \varphi$ we have $s=0$ and $a=d\left(\ln \sqrt{-g_{t t}}\right)$ and then the function $U$ will be $U=\ln \sqrt{-g_{t t}}$, such that $U_{\mid \Sigma}=0$ (rigid rotating virtual fluid), so we have a $\theta_{\text {out }}^{0}$ satisfying the conditions $\theta_{i n \mid \Sigma}^{0}=\theta_{o u t \mid \Sigma}^{0}$ and $w \wedge s=0$. However, in this situation this choice is not unique; for instance, 
another possibility is $\theta^{0}=\left(-\left(g_{t t} g_{\varphi \varphi}-g_{t \varphi}^{2}\right) / g_{\varphi \varphi}\right)^{1 / 2} d t$, $\theta^{1}=\sqrt{g_{\varphi \varphi}}\left(d \varphi+\left(g_{t \varphi} / g_{\varphi \varphi}\right) d t\right) \quad$ where $U$ $=\ln \sqrt{-\left(g_{t t} g_{\varphi \varphi}-g_{t \varphi}^{2}\right) / g_{\varphi \varphi}}$ which also satisfies the imposed conditions (virtual fluid with irrotational motion).

Hence, we can always choose a $\theta^{0}$ in the exterior region satisfying $w \wedge s=0$ and coinciding with a prescribed one in $\Sigma$, which is a "static fluid surface" [24]. In fact, it is possible to find several ones, but, for instance, we can always choose the virtual fluid with irrotational motion. Therefore, we have reduced this problem to a two-fluid problem, except that outside we have $p=0$ and $\mu=0$.

\section{B. Matching known solutions}

We have analyzed the problem of obtaining new interior and new exterior solutions. Now, let us analyze the theoretical problem when one (the interior or the exterior) or both solutions are known.

Suppose that an exact interior solution is known. The matching hypersurface is given by the equation $p=0$. It is possible to introduce $U$ and $v$ coordinates in this interior region. Then, we can formulate the boundary problem for the exterior region using the $U$ and $v$ coordinates and the parametrization presented in Sec. IV. This is implemented by giving the values of $G$ and $\varpi$ and their $U$ derivatives in the matching surface $U=0$.

Let us consider the case when an exterior exact solution is known in some coordinates, say $x$ and $v$. First, we have to choose a matching hypersurface $\Sigma$, given by an implicit equation $F(x, v)=0$. Starting from a rigid rotating tetrad with caret for the asymptotic $t$ and $\varphi$ coordinates, we can write down the PDE master equation. The boundary problem for this equation is fixed by giving a $\lambda(v)$ in $\Sigma$, which is equivalent to fixing a fluid angular velocity distribution on $\Sigma$ (with respect to a non-rotating observer at infinity). This also fixes the function $h(\varpi)$ on $\Sigma$. The matching conditions determine $h(\varpi)$ in the interior, at least locally around $\Sigma$. For a perfect fluid with a given barotropic equation of state $\mu$ $=\mu(p)$ we can formulate a boundary problem for the interior region in $U$ and $v$ coordinates.

When we know an exact interior solution and an exact exterior solution, we have to look for the matching surface $\Sigma$, which will be given by an equation $F(x, v)=0$ in the exterior and by $p=0$ in the interior. On $\Sigma$ we have $\theta_{\mid \Sigma}^{0}$ invariantly defined by the interior fluid velocity, then the PDE master equation and its boundary condition determine $\theta_{\text {out }}^{0}$, and the $U$ and $v$ coordinates can be introduced in the exterior, at least locally around $\Sigma$. The matching conditions presented in Sec. IV, will determine if there is a hypersurface $\Sigma$ where the two spacetimes can be matched.

\section{MATCHING INTERIOR AND EXTERIOR SCHWARZSCHILD SOLUTION IN $U$ AND $v$ COORDINATES}

In this section, we will present a very simple example of the matching in $U$ and $v$ coordinates; in particular, we will apply the formalism for the case of a static solution consist- ing of the interior Schwarchild solution ( $\mu=$ const) and the exterior Schwarchild solution.

\section{A. Interior Schwarzschild solution}

The line element of this solution can be written as follows:

$$
\begin{aligned}
d s^{2}= & -\left(a-b \sqrt{q-\frac{r^{2}}{R^{2}}}\right)^{2} d t^{2}+\frac{1}{1-\frac{r^{2}}{R^{2}}} d r^{2} \\
& +r^{2}\left(d \theta^{2}+\sin ^{2} \theta d \varphi^{2}\right)
\end{aligned}
$$

where $a, b, q$, and $R$ are constants. This solution represents a perfect fluid in comoving coordinates and with equation of state $\mu=$ const [23]. We can choose an orthonormal tetrad following the prescription described above $\left(\theta^{0}\right.$ is the velocity of the fluid and $\theta^{1}$ is orthogonal to $\theta^{0}$ in the $\{t, \varphi\}$ subspace)

$$
\begin{aligned}
& \theta^{0}=\left(a-b \sqrt{1-\frac{r^{2}}{R^{2}}}\right) d t \\
& \theta^{1}=r \sin \theta d \varphi,
\end{aligned}
$$

for the kinematical properties we have

$$
\begin{aligned}
& a=d \ln \left(a-b \sqrt{1-\frac{r^{2}}{R^{2}}}\right) \\
& b=d \ln \left[\left(a-b \sqrt{1-\frac{r^{2}}{R^{2}}}\right) r \sin \theta\right]
\end{aligned}
$$

and $w$ and $s$ vanish. Then, the change to $U$ and $v$ coordinates is described by the following relations:

$$
\begin{gathered}
U=\ln \left(a-b \sqrt{1-\frac{r^{2}}{R^{2}}}\right)+U_{0}, r=R \sqrt{1-\left(\frac{e^{U-U_{0}-a}}{b}\right)^{2}} \\
v=\ln \left[\left(a-b \sqrt{\left.1-\frac{r^{2}}{R^{2}}\right)} r \sin \theta\right]+v_{0},\right. \\
\sin \theta=\frac{e^{-\left(U-U_{0}\right)} e^{v-v_{0}}}{R \sqrt{1-\left(\frac{e^{U-U_{0}-a}}{b}\right)^{2}}} .
\end{gathered}
$$

The line element in these coordinates can be written as 


$$
\begin{aligned}
d s^{2}= & -e^{2\left(U-U_{0}\right)} d t^{2}+e^{-2\left(U-U_{0}\right)} e^{2\left(v-v_{0}\right)} d \varphi^{2}+R^{2} \frac{e^{2\left(U-U_{0}\right)}}{b^{2}-\left(e^{\left(U-U_{0}\right)}-a\right)^{2}} d U^{2} \\
& +\frac{\left\{d v+\left[-1+\frac{\left(e^{\left(U-U_{0}\right)}-a\right) e^{\left(U-U_{0}\right)}}{b^{2}-\left(e^{\left(U-U_{0}\right)}-a\right)^{2}}\right] d U\right\}^{2}}{e^{2\left(U-U_{0}\right)} e^{-2\left(v-v_{0}\right)}-\frac{b^{2}}{R^{2}\left[b^{2}-\left(e^{\left(U-U_{0}\right)}-a\right)^{2}\right]}}
\end{aligned}
$$

and $\theta^{0}$ and $\theta^{1}$ in these coordinates read as follows:

$$
\begin{aligned}
& \theta^{0}=e^{U-U_{0}} d t \\
& \theta^{1}=e^{-\left(U-U_{0}\right)} e^{\left(v-v_{0}\right)} d \varphi .
\end{aligned}
$$

The pressure in these coordinates reads

$$
p=\frac{-3+2 a e^{U_{0}-U}}{R^{2}}
$$

and the energy density is a constant $\mu=3 / R^{2}$.

\section{B. Exterior Schwarzschild solution}

The Schwarzschild spacetime for vacuum in the standard coordinates can be written as follows:

$$
d s^{2}=-\left(1-\frac{2 m}{r}\right) d t^{2}+\frac{1}{1-\frac{2 m}{r}} d r^{2}+r^{2}\left(d \theta^{2}+\sin ^{2} \theta d \varphi^{2}\right) .
$$

Following our approach, we have to choose a tetrad such that $w \wedge s=0$ and $\theta_{i n \mid \Sigma}^{0}=\theta_{o u t \mid \Sigma}^{0}$. We can start with a tetrad of the form

$$
\begin{aligned}
& \widehat{\theta^{0}}=\sqrt{1-\frac{2 m}{r}} d t \\
& \widehat{\theta^{1}}=r \sin \theta d \varphi
\end{aligned}
$$

and look for the required transformation. Hence, we have to solve the differential form master equation (41) that in this case reduces to

$$
d \lambda \wedge \frac{\sinh \lambda}{\cosh \lambda} \hat{\beta}=0
$$

from this equation we have $\lambda=$ const or

$$
2 d \lambda=\alpha \frac{\sinh \lambda}{\cosh \lambda} \hat{\beta}
$$

where $\alpha \neq 0$. For our case this equation can be decomposed into

$$
\alpha=2 \tan \theta \frac{\lambda_{\theta}}{\sinh (2 \lambda)}
$$

and

$$
\frac{r(r-2 m)}{r-3 m} \lambda_{r}=\tan \theta \lambda_{\theta}
$$

This first-order partial differential equation has the general solution

$$
\lambda=F\left(\frac{\sqrt{r-2 m}}{r^{3 / 2} \sin \theta}\right)
$$

where $F$ is an arbitrary function of its argument. Let us mention that the general solution of the corresponding first-order partial differential equation can be obtained in the case of a general static spherically symmetric spacetime

$$
d s^{2}=-e^{2 \nu(r)} d t^{2}+e^{2 \beta(r)} d r^{2}+r^{2}\left(d \theta^{2}+\sin ^{2} \theta d \varphi^{2}\right)
$$

where we have to solve the equation

$$
\frac{1}{\frac{1}{r}-\nu^{\prime}} \lambda_{r}=\tan (\theta) \lambda_{\theta}
$$

the solution in this case is $\lambda=F\left(e^{-\nu} r \sin \theta\right)$ where $F$ is an arbitrary function of its argument.

Now, suppose that we impose the condition that $\theta_{i n \mid \Sigma}^{0}$ $=\theta_{\text {out } \mid \Sigma}^{0}$, assuming that $\lambda$ is not a constant. We have

$$
\theta_{i n}^{0}=\left(a-b \sqrt{1-\frac{r^{2}}{R^{2}}}\right) d \hat{t}
$$

and

$$
\begin{aligned}
\theta_{\text {out }}^{0}= & \cosh \left(F\left(\frac{\sqrt{r-2 m}}{r^{3 / 2} \sin \theta}\right)\right) \sqrt{1-\frac{2 m}{r}} d t \\
& +\sinh \left(F\left(\frac{\sqrt{r-2 m}}{r^{3 / 2} \sin \theta}\right)\right) r \sin \theta d \varphi .
\end{aligned}
$$

If $\hat{t}=c_{1} t+c_{2} \varphi$, we get 


$$
\theta_{i n}^{0}=c_{1}\left(a-b \sqrt{1-\frac{r^{2}}{R^{2}}}\right) d t+c_{2}\left(a-b \sqrt{1-\frac{r^{2}}{R^{2}}}\right) d \varphi,
$$

but then from the coefficient of $d t$ we should have $F$ $=$ const, against our assumption. Now, let us consider the case with $\lambda=\lambda_{0}$ (a constant). We have

$$
\theta_{\text {out }}^{0}=\cosh \left(\lambda_{0}\right) \sqrt{1-\frac{2 m}{r}} d t+\sinh \left(\lambda_{0}\right) r \sin \theta d \varphi
$$

and

$$
\theta_{i n}^{0}=c_{1}\left(a-b \sqrt{1-\frac{r^{2}}{R^{2}}}\right) d t+c_{2}\left(a-b \sqrt{1-\frac{r^{2}}{R^{2}}}\right) d \varphi
$$

in order to have $\theta_{i n \mid \Sigma}^{0}=\theta_{\text {out } \mid \Sigma}^{0}$, we should have $c_{2}=0$ and $\lambda_{0}=0$, also we can choose $c_{1}=1$, that implies $\hat{t}=t$ (we assume $\hat{\varphi}=\varphi$ ). Hence, we have that the required tetrad elements are

$$
\begin{aligned}
& \theta^{0}=\sqrt{1-\frac{2 m}{r}} d t \\
& \theta^{1}=r \sin \theta d \varphi .
\end{aligned}
$$

For these tetrad elements we have

$$
\begin{aligned}
& a=d \ln \left(\sqrt{1-\frac{2 m}{r}}\right)=d U \\
& b=d \ln \left(\sqrt{1-\frac{2 m}{r}} r \sin \theta\right)=d v,
\end{aligned}
$$

and $w$ and $s$ vanish. The change to $\{U, v\}$ coordinates is given by the following relations:

$$
\begin{gathered}
U=\frac{1}{2} \ln \left(1-\frac{2 m}{r}\right)+U_{0}, \quad r=\frac{2 m}{1-e^{2\left(U-U_{0}\right)}} \\
v=\frac{1}{2} \ln \left[\left(1-\frac{2 m}{r}\right) r^{2} \sin ^{2} \theta\right]+v_{0}, \\
\sin \theta=-\frac{1}{m} e^{v-v_{0}} \sinh \left(U-U_{0}\right) .
\end{gathered}
$$

The line element in those coordinates can be written as

$$
\begin{aligned}
d s^{2}= & -e^{2\left(U-U_{0}\right)} d t^{2}+e^{-2\left(U-U_{0}\right)} \\
& \times\left\{e^{2\left(v-v_{0}\right)} d \varphi^{2}+\frac{m^{2}}{\sinh ^{4}\left(U-U_{0}\right)} d U^{2}\right. \\
& +\frac{m^{2}}{e^{-2\left(v-v_{0}\right)} m^{2}-\sinh ^{2}\left(U-U_{0}\right)} \\
& \left.\times\left[d v+\frac{\cosh \left(U-U_{0}\right)}{\sinh \left(U-U_{0}\right)} d U\right]^{2}\right\}
\end{aligned}
$$

and $\theta^{0}$ and $\theta^{1}$ in these coordinates read as follows:

$$
\begin{aligned}
& \theta^{0}=e^{U-U_{0}} d t \\
& \theta^{1}=e^{-\left(U-U_{0}\right)} e^{\left(v-v_{0}\right)} d \varphi .
\end{aligned}
$$

The condition that $\theta_{\text {in }}^{0}=\theta_{\text {out }}^{0}$ in $U=0$, as well as the definition of $\theta^{1}$, impose that $U_{0, \text { in }}=U_{0, \text { out }}$ and $v_{0, \text { in }}=v_{0, \text { out }}$. The matching conditions are the continuity of the functions $f, G, N$, and $G_{U}$; as $\varpi_{U}, \varpi_{v}$, and $h(\varpi)$ vanish. The continuity of $f, G$, and $N$ impose the following relations:

$$
m^{2}=\frac{R^{2}}{b^{2}} e^{-2 U_{0}}\left[b^{2}-\left(e^{-U_{0}}-a\right)^{2}\right] \sinh ^{2} U_{0}
$$

$$
\begin{gathered}
\frac{m^{2} e^{2 U_{0}}}{\sinh ^{4} U_{0}}=\frac{R^{2} e^{-2 U_{0}}}{b^{2}-\left(e^{\left.-U_{0}-a\right)^{2}}\right.} \\
-\frac{\cosh U_{0}}{\sinh U_{0}}=-1+\frac{\left(e^{-U_{0}-a}\right) e^{-U_{0}}}{b^{2}-\left(e^{-U_{0}}-a\right)^{2}} .
\end{gathered}
$$

The solution of these equations is

$$
\begin{aligned}
e^{-U_{0}} & =\frac{2}{3} a \\
b^{2} & =1 / 4 \\
m & =\frac{R}{2}\left(1-\frac{4}{9} a^{2}\right)^{3 / 2} .
\end{aligned}
$$

By substituting these relations in the condition imposed by the continuity of $G_{U}$ we have that this condition is identically satisfied (in fact, this happens for any static spherically symmetric spacetime). In order to compare the results with those obtained by the matching procedure in $r$ and $\theta$ coordinates, we use that $U=0$ implies that $r=r_{0}$, and then we have $e^{-U_{0}}=\sqrt{1-2 m / r_{0}} \quad$ or, what is equivalent, $a$ $=\frac{3}{2} \sqrt{1-2 m / r_{0}}$, and then $m=r_{0}^{3} / 2 R^{2}$ and $a=\frac{3}{2} \sqrt{1-r_{0}^{2} / R^{2}}$. These are the relations obtained from a direct matching in $r$ and $\theta$ coordinates.

In Fig. 1 we plot the interior (filled) and the exterior regions of the Schwarzschild spacetimes in $U$ and $v$ coordinates. The two regions are bounded by two asymptotes of $U=$ const and the equatorial plane curve. Note that the $r$ $=$ const lines corresponds to $U=$ const, in particular, the boundary of the star corresponds to $U=0$, the asymptote with the lower value of $U$ represents the curve $r=0$, and $r$ $=\infty$ is the asymptote with the upper value of $U$. The curved boundary of the regions corresponds to the line $\theta=\pi / 2$ (the equatorial plane) and the other curves inside the regions represent $\theta=$ const for different values of the constant. The region is doubly covered by curves of $\theta=$ const as a consequence of the symmetry of the solution with respect to the equatorial plane $(\theta=\pi / 2)$. 


\section{THE PDE MASTER EQUATION FOR THE KERR METRIC}

As an example of the first steps towards matching a vacuum exterior solution with an interior perfect fluid, let us study the PDE master equation for the Kerr metric. We write the metric in Boyer-Linquist coordinates [22]

$$
\begin{aligned}
d s^{2}= & -d t^{2}+\left(r^{2}+a^{2}\right) \sin ^{2} \theta d \varphi^{2}+\frac{2 m r}{\Sigma}\left(a \sin ^{2} \theta d \varphi-d t\right)^{2} \\
& +\Sigma\left(\frac{d r^{2}}{\Delta}+d \theta^{2}\right)
\end{aligned}
$$

where $\Sigma=r^{2}+a^{2} \cos ^{2} \theta$ and $\Delta=r^{2}-2 m r+a^{2}$. We take a "rigid rotating" tetrad ( $s=0 \Leftrightarrow \delta=\kappa=w)$ as the starting tetrad (with caret)

$$
\begin{aligned}
& \widehat{\theta^{0}}=\frac{\mathcal{A}}{\mathcal{B}}\left(d t+\frac{\mathcal{C}}{\mathcal{A}^{2}} d \varphi\right) \\
& \widehat{\theta^{1}}=\frac{\mathcal{B D}}{\mathcal{A}} d \varphi
\end{aligned}
$$

where

$$
\begin{aligned}
& \mathcal{A}=\left(r^{2}-2 m r+a^{2} \cos ^{2} \theta\right)^{1 / 2} \\
& \mathcal{B}=\Sigma^{1 / 2} \\
& \mathcal{C}=2 a m r \sin ^{2} \theta \\
& \mathcal{D}=\Delta^{1 / 2} \sin \theta,
\end{aligned}
$$

then the first-order partial differential equation defining the transformations maintaining the property that $w \wedge s=0$ (i.e. $a=d U)$ is

$$
\frac{2 \lambda_{, r}+\hat{\kappa}_{r}}{\sinh (2 \lambda) \hat{\beta}_{r}+\cosh (2 \lambda) \hat{\delta}_{r}}=\frac{2 \lambda_{, v}+\hat{\kappa}_{v}}{\sinh (2 \lambda) \hat{\beta}_{v}+\cosh (2 \lambda) \hat{\delta}_{v}}
$$

where the $v$ coordinate is defined by $v=\ln D+v_{0}$ $=\ln \left(\Delta^{1 / 2} \sin \theta\right)+v_{0}$ and

$$
\begin{aligned}
\widehat{\delta_{v}}=\widehat{\kappa_{v}} & =\frac{4 a m r \Delta^{2} x^{3}}{\Omega} \\
\widehat{\delta_{r}}=\widehat{\kappa_{r}} & =\frac{2 a m \Delta\left(-3 r^{2}+2 m r+a^{2}\right) x^{3}}{\Omega} \\
\widehat{\beta_{v}} & =\frac{\Gamma \Delta^{3} x^{4}-2 a^{2}\left(r^{2}-3 m r+a^{2}\right)}{\Omega} \\
\widehat{\beta_{r}} & =\frac{2 m\left(a^{2}-r^{2}\right) \Delta^{2} x^{4}-2 m a^{2}(3 r}{\Omega} \\
\Omega & =\Gamma \Delta^{3} x^{4}-a^{2} \Delta(\Gamma+\Delta) x^{2}+a^{4} \\
\Gamma & =a^{2}+r^{2} \\
x & =e^{-\left(v-v_{0}\right)} .
\end{aligned}
$$$$
\widehat{\delta_{r}}=\widehat{\kappa_{r}}=\frac{2 a m \Delta\left(-3 r^{2}+2 m r+a^{2}\right) x^{3}-2 a^{3} m x}{\Omega}
$$$$
\widehat{\beta_{v}}=\frac{\Gamma \Delta^{3} x^{4}-2 a^{2}\left(r^{2}-3 m r+a^{2}\right) \Delta x^{2}+a^{4}}{\Omega}
$$$$
\widehat{\beta_{r}}=\frac{2 m\left(a^{2}-r^{2}\right) \Delta^{2} x^{4}-2 m a^{2}\left(3 r^{2}-4 m r+a^{2}\right) x^{2}}{\Omega}
$$

We are not able to find the general solution for this equation, but we found a particular solution

$$
\lambda=\operatorname{arcsinh}\left(\frac{2 m a r \sin ^{2} \theta\left(r^{2}+a^{2} \cos ^{2} \theta-2 m r\right)^{-1 / 2}}{\sqrt{\left[r^{4}+a^{2} r^{2}\left(\cos ^{2} \theta+1\right)+2 m r a^{2} \sin ^{2} \theta+a^{4} \cos ^{2} \theta\right] \sin ^{2} \theta}}\right) .
$$

The matching surface for this particular solution is given by the implicit equation

$$
\frac{\left(r^{2}+a^{2} \cos ^{2} \theta\right)\left(r^{2}-2 m r+a^{2}\right)}{\left(r^{2} a^{2}-2 m r a^{2}+a^{4}\right) \cos ^{2} \theta+r^{4}+r^{2} a^{2}+2 m r a^{2}}=k,
$$

where $k=$ const. This solution corresponds to the case when the interior perfect fluid is in irrotational motion, at least near the boundary.

In Fig. 2 we present a plot of the function $\lambda$ for $a=1.9$, $m=2$. The height in the plot represents the value of $\lambda$ and the other two coordinates are $z=r \cos \theta$ and $\rho=r \sin \theta$ (applying axial symmetry, we plot together $\varphi=0$ and $\varphi=\pi$ for clarity, and we use the coordinate $X=\rho \cos \varphi$ ). In Fig. 3, using the same coordinates, we plot the level surfaces for $\lambda$, the matching surface for $k=0.1$ (the broader solid line), the surface $r=$ const inscribed in the boundary (gray filled region), the ergoregion (the doughnut-like shaped region), and the horizon (the $r=$ const surface inscribed in the ergoregion) for $a=1.9$ and $m=2$. We can see that, for this case, the level surfaces of $\lambda$ have a dipole-like form such that on the boundary surface the value of $\lambda$ decreases from the equator to the poles. Also, we can check that the part of the boundary nearest to the poles is outside the ergosphere but the part around the equator is included in the ergosphere. Note that the horizon is inside the boundary and would be completely covered by the fluid.

The change to $U$ and $v$ coordinates is the following: 


$$
\begin{aligned}
U-U_{0} & =\frac{1}{2} \ln \left(\frac{\left(r^{2}+a^{2} \cos ^{2} \theta\right)\left(r^{2}-2 m r+a^{2}\right)}{r^{4}+r^{2} a^{2}+2 a^{2} m r+\left(r^{2} a^{2}-2 m r a^{2}+a^{4}\right) \cos ^{2} \theta}\right) \\
v-v_{0} & =\frac{1}{2} \ln \left[\left(r^{2}-2 m r+a^{2}\right) \sin ^{2} \theta\right] .
\end{aligned}
$$

In Fig. 4 we plot the Kerr exterior region in $U$ and $v$ coordinates for $a=1.9$ and $m=2$. The similarity with Fig. 1 corresponding to the Schwarzschild solution is apparent.

\section{A particular solution for the PDE master equation for a generic spacetime}

After we found a particular solution for the PDE master equation for the Kerr metric we thought that perhaps it should be possible to find a particular solution for the PDE master equation for a generic spacetime.

For a generic stationary and axisymmetric spacetime we can always choose "rigid rotating" tetrad elements as follows:

$$
\begin{aligned}
& \widehat{\theta^{0}}=\sqrt{-g_{t t}}\left(d t+\frac{g_{t \varphi}}{g_{t t}} d \varphi\right) \\
& \widehat{\theta^{1}}=\sqrt{\frac{g_{t \varphi}^{2}-g_{\varphi \varphi} g_{t t}}{-g_{t t}}} d \varphi .
\end{aligned}
$$

For these tetrad elements we have that

$$
\begin{aligned}
& \hat{b}=d \ln \sqrt{g_{t \varphi}^{2}-g_{\varphi \varphi} g_{t t}} \\
& \hat{a}=d \ln \sqrt{-g_{t t}} . \\
& \hat{w}=-\frac{-g_{t t}}{\sqrt{g_{t \varphi}^{2}-g_{\varphi \varphi} g_{t t}}} d\left(\frac{g_{t \varphi}}{g_{t t}}\right) \\
& \hat{s}=0
\end{aligned}
$$

then

$$
\begin{gathered}
\hat{\delta}=\hat{\kappa}=\hat{w}=-\frac{-g_{t t}}{\sqrt{g_{t \varphi}^{2}-g_{\varphi \varphi} g_{t t}}} d\left(\frac{g_{t \varphi}}{g_{t t}}\right) \\
\hat{b}-2 \hat{a}=d \ln \left(\frac{\sqrt{g_{t \varphi}^{2}-g_{t t} g_{\varphi \varphi}}}{-g_{t t}}\right) .
\end{gathered}
$$

The first-order PDE master equation determining the gauge transformations that maintains the property that $\delta \wedge \kappa$ $=0 \Leftrightarrow d a=0$ can be written easily in terms of these expressions. To obtain the general solution of this master equation is a formidable problem, however we always know a particular solution which is given by the following expression:

$$
\lambda=\operatorname{arcsinh}\left(\frac{g_{t \varphi}}{\sqrt{-g_{t t} g_{\varphi \varphi}}}\right) .
$$

This solution corresponds to passing from a "rigid rotating" tetrad to an "irrotational motion tetrad," which is always possible. For any stationary and axisymmetric metric we can choose

$$
\begin{aligned}
\theta^{0} & =\sqrt{\frac{g_{t \varphi}^{2}-g_{t t} g_{\varphi \varphi}}{g_{\varphi \varphi}}} d t \\
\theta^{1} & =\sqrt{g_{\varphi \varphi}}\left(d \varphi+\frac{g_{t \varphi}}{g_{\varphi \varphi}} d t\right),
\end{aligned}
$$

and then

$$
\begin{aligned}
& b=d \ln \sqrt{g_{t \varphi}^{2}-g_{\varphi \varphi} g_{t t}} \\
& a=d \ln \sqrt{\frac{g_{t \varphi}^{2}-g_{t t} g_{\varphi \varphi}}{g_{\varphi \varphi}}} \\
& s=\frac{g_{\varphi \varphi}}{\sqrt{g_{t \varphi}^{2}-g_{\varphi \varphi} g_{t t}}} d\left(\frac{g_{t \varphi}}{g_{\varphi \varphi}}\right) \\
& w=0 .
\end{aligned}
$$

The matching surface in this case will be given by

$$
\frac{g_{t \varphi}^{2}-g_{t t} g_{\varphi \varphi}}{g_{\varphi \varphi}}=k
$$

where $k$ is a constant. The previous solution for $\lambda$ will be determined in a unique manner by the value of $\lambda$ in the surface given by Eq. (119).

\section{CONCLUSIONS}

We have presented the matching conditions for two rotating fluids in terms of the kinematical properties of both fluids in general relativity. A simplified form of the Einstein equations has been given, using coordinates adapted to the hypersurface separating both fluids. A physical situation where this analysis can be used is the case of a rotating relativistic star with two regions with different physical properties, equations of state, rotation laws, . . , separated by a well-defined surface. The two-fluid problem is reduced to a system of two nonlinear second-order partial differential equation in two variables for two functions in the interior and exterior re- 


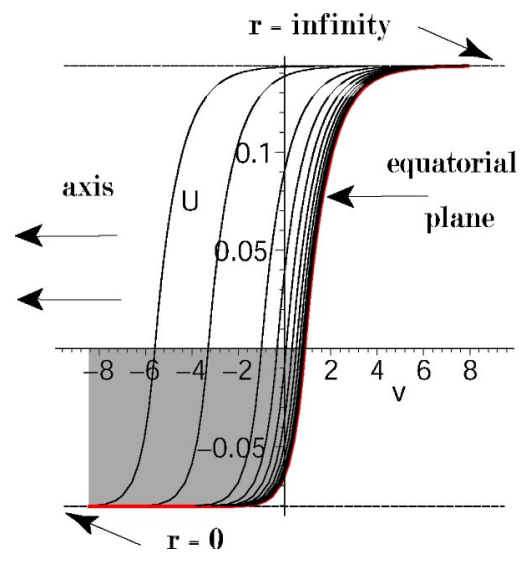

FIG. 1. Interior and exterior regions of Schwarzschild spacetimes in $U$ and $v$ coordinates.

1gions. The boundary conditions for these functions are the continuity of them and their $U$ derivatives on the matching surface, which is described by the equation $U=0$.

The matching of a perfect fluid interior region and an exterior vacuum region is also analyzed as a model for an isolated rotating relativistic star (the models for the interior and exterior regions should be calculated at the same time). By solving a boundary problem for a first order partial differential equation (master equation), a tetrad is defined for the exterior region such that the matching (Darmois) and Einstein equations reproduce those of the two-fluid problem. Therefore, this problem can also be reduced to a boundary problem for a system of two nonlinear second order partial differential equations in two variables for two functions in both regions.

A static spherically symmetric star described by the interior and exterior Schwarzschild solutions is analyzed within formalism. The Kerr metric is also studied, the master equation written, some particular exact solutions found, and the change to coordinates $U$ and $v$ for those solutions described.

Two problems currently under consideration are the asymptotic flatness in $U$ and $v$ coordinates and the equatorial symmetry; for both problems the analysis of the spherically symmetric model, presented in Sec. IV, can be used as a guide. Also, we would like to mention that, in principle, it is possible to develop a numerical code using coordinates adapted to the surface of the star, or a transition surface, using the formalism and results developed in this paper to produce a complementary approach to the already existing codes.

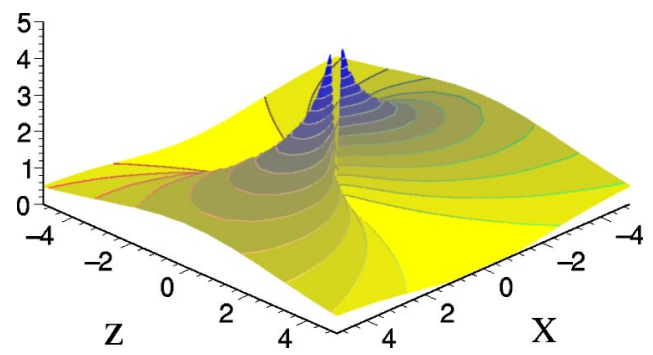

FIG. 2. 3D plot of $\lambda$ for the Kerr metric.

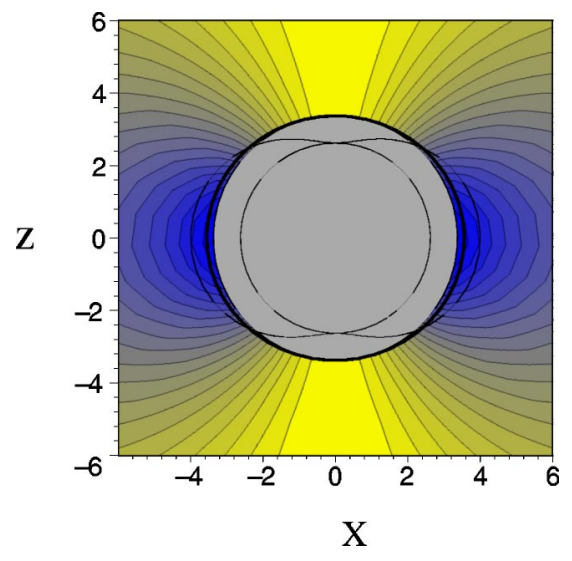

FIG. 3. Level surfaces of $\lambda$ for the Kerr metric.

\section{ACKNOWLEDGMENTS}

The present work has been supported in part by DGICYT Project PB98-0772. The author wishes to thank F. J. Chinea, L. Fernández-Jambrina, F. Navarro-Lérida and M. J. Pareja for valuable discussions.

\section{APPENDIX: DIFFERENTIAL FORM APPROACH SUMMARY}

We consider a stationary and axisymmetric spacetime describing a perfect fluid in circular motion. That means that it admits two Killing fields $\{\xi, \eta\}, \xi$ time-like and $\eta$ space-like also $[\xi, \eta]=0$ and $u$ (the velocity of the fluid) belongs to $\operatorname{Lin}\{\xi, \eta\}$.

Here we present a summary of a differential form approach for this problem; see [20] for more details. We use an orthonormal tetrad

$$
d s^{2}=-\theta^{0} \otimes \theta^{0}+\theta^{1} \otimes \theta^{1}+\theta^{2} \otimes \theta^{2}+\theta^{3} \otimes \theta^{3}
$$

where $\theta^{0} \equiv u$ and $\theta^{0}$ and $\theta^{1}$ are in the space of the orbits of the symmetry group. We choose a gauge where the Lie derivative of all the tetrad elements $\theta^{i}, i=0,1,2,3$ in the direction of $\xi$ and $\eta$ vanishes (so no function appearing in the metric will depend on the coordinates adapted to the Killing fields). We introduce several differential 1 -forms $a, w, s, b$, and $\nu$ all in $\operatorname{Lin}\left\{\theta^{2}, \theta^{3}\right\}$ where $a$ is the acceleration of the fluid velocity, $w$ is a 1-form such that the vorticity tensor can be written as $\omega_{T}=\theta^{1} \wedge w, s$ is a 1-form such that the shear tensor is $S_{T}=\theta^{1} \otimes_{s} s$, b is the derivative of the logarithm of the determinant of the metric in the $\{\xi, \eta\}$ subspace, and $\nu$ is the Riemannian connection in the $\left\{\theta^{2}, \theta^{3}\right\}$ subspace. After some simplification using a gauge fixing (we impose $\theta^{2}$ $\propto b$ ) we find the following exterior differential system which is equivalent to the vanishing torsion equations, the first $\mathrm{Bi}$ anchi identities, the Einstein equations, and the second Bianchi identities:

$$
\begin{aligned}
d u & =a \wedge u+w \wedge \theta^{1} \\
d \theta^{1} & =(b-a) \wedge \theta^{1}+s \wedge u \\
d b & =0
\end{aligned}
$$




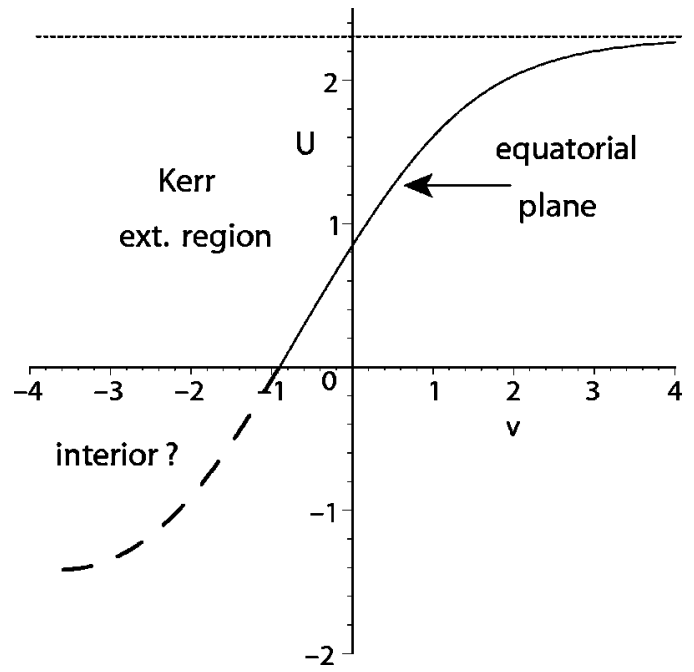

FIG. 4. Kerr metric in $U$ and $v$ coordinates.

$$
\begin{gathered}
d a=w \wedge s \\
d w=-(b-2 a) \wedge w \\
d s=(b-2 a) \wedge s \\
d \star(w-s)=-2 a \wedge \star w-2(a-b) \wedge \star s \\
d \star b+b \wedge \star b=2 p \frac{b \wedge \star b}{|b|^{2}} \\
d \star a+b \wedge \star a=-\frac{1}{2} w \wedge \star w+\frac{1}{2} s \wedge \star s \\
+\frac{1}{2}(\mu+3 p) \frac{b \wedge \star b}{|b|^{2}} \\
-\frac{1}{2} d\left(|b|^{2}\right)=\left(|b|^{2}-p\right) b-|b|^{2} a+[\langle b, a\rangle a \\
-\langle b, \star a\rangle \star a]-\frac{1}{4}[\langle b,(s-w)\rangle \quad(s-w) \\
-\langle b, \star(s-w)\rangle \star(s-w)] \\
d p=-(\mu+p) a
\end{gathered}
$$

where $\mu$ is the energy density of the fluid, $p$ is the pressure, $\star$ stands for the Hodge dual in the $\left\{\theta^{2}, \theta^{3}\right\}$ subspace, \langle\rangle indicates the scalar product, and || means the norm. This is the system of equations we have to solve. We have the following expression for $\nu$ :

$$
\nu=\frac{1}{2} \star \frac{d\left(|b|^{2}\right)}{|b|^{2}}+\left(1-2 \frac{p}{|b|^{2}}\right) \star b .
$$

In the present paper we use a parametrization where $|b|^{2} \equiv 1 / G,|a|^{2}=N|b|^{2}$, and $\langle b, a\rangle=-f|b|^{2}$.

When $d a=0$ we have $w \wedge s=0$ and, using the vanishing torsion equations, we can parametrize $w$ and $s$ as follows:

$$
\begin{aligned}
w & =\hat{\kappa} e^{\xi} d \varpi \\
s & =\hat{\epsilon} e^{-\xi} d \varpi,
\end{aligned}
$$

where $\xi=2 U-v+2 h(\varpi), a=d U, b=d v, \kappa^{2}=1,0$, and $\epsilon^{2}=1,0$.

Then $\theta^{0}$ and $\theta^{1}$ can be parametrized as

$$
\begin{aligned}
& \theta^{0}=e^{U}[A d t+B d \varphi] \\
& \theta^{1}=e^{v-U}[C d t+D d \varphi],
\end{aligned}
$$

where $A, B, C$, and $D$ are functions of $\varpi$ that have to satisfy the following linear system of ordinary differential equations:

$$
\frac{d S}{d \varpi}=\left(\begin{array}{cc}
0 & \kappa e^{2 h(\varpi)} \\
\epsilon e^{-2 h(\varpi)} & 0
\end{array}\right) S, \quad S \equiv\left(\begin{array}{ll}
A & B \\
C & D
\end{array}\right) .
$$

Therefore, if the rotation regime is fixed by means of a particular function $h(\varpi)$ we can obtain, by solving this linear ordinary differential system, $A=A(\varpi), \quad B=B(\varpi), \quad C$ $=C(\varpi)$, and $D=D(\varpi)$.

The determinant of $S$ is constant: $(d / d \varpi)(A D-B C)$ $=0$; if $S$ is a solution then $S^{\prime} \equiv S P$ is also a solution, whenever $P$ is a constant matrix; this freedom is nothing else but the freedom of choice of the $t$ and $\varphi$ coordinates; if $t$ and $\varphi$ transform according to the relations

$$
\begin{aligned}
t^{\prime} & =k_{1} t+k_{2} \varphi \\
\varphi^{\prime} & =k_{3} t+k_{4} \varphi
\end{aligned}
$$

(where $k_{1}, k_{2}, k_{3}$, and $k_{4}$ are constants), then the corresponding $P$ is given by

$$
P^{-1}=\left(\begin{array}{ll}
k_{1} & k_{2} \\
k_{3} & k_{4}
\end{array}\right)
$$

When $f, G, N$, and $\varpi$ are known as functions of $U$ and $v$, the metric can be written as follows:

$$
\begin{aligned}
d s^{2}= & -e^{2 U}[A(\varpi) d t+B(\varpi) d \varphi]^{2}+e^{2 v-2 U} \\
& \times[C(\varpi) d t+D(\varpi) d \varphi]^{2} \\
+ & \frac{G}{N-f^{2}}\left[N d v^{2}+d U^{2}+2 f d U d v\right] .
\end{aligned}
$$

[1] G. Darmois, Mémorial des Sciences Mathématiques (GauthierVillars, Paris, 1927), Vol. 25.

[2] A. Lichnerowicz, Théories Relativistes de la Gravitation et de l'Electromagnétism (Masson, Paris, 1955).
[3] S. O'Brien and J.L. Synge, Comm. Dublin Inst. Adv. Stud. Ser. A 9, 1 (1952).

[4] W. Israel, Nuovo Cimento B B44, 1 (1966).

[5] W.B. Bonnor and P.A. Vickers, Gen. Relativ. Gravit. 13, 29 
(1981)

[6] L. Herrera and J. Jimenéz, Phys. Rev. D 28, 2987 (1983).

[7] A.H. Taub, J. Math. Phys. 21, 1423 (1979).

[8] C.J.S. Clarke and T. Dray, Class. Quantum Grav. 4, 265 (1987).

[9] C. Barrabes and W. Israel, Phys. Rev. D 43, 1129 (1991); C. Barrabes, Class. Quantum Grav. 6, 581 (1989).

[10] M. Mars and J.M.M. Senovilla, Class. Quantum Grav. 10, 1865 (1993).

[11] R. Vera, Class. Quantum Grav. 19, 5249 (2002).

[12] G. Neugebauer and R. Meinel, Phys. Rev. Lett. 73, 2166 (1994).

[13] M. Mars and J.M.M. Senovilla, Mod. Phys. Lett. A A 13, 1509 (1998).

[14] M. Bradley, G. Fodor, M. Marklud, and Z. Perjés, Class. Quantum Grav. 17, 351 (2000).

[15] E. Kyriakopoulos, Gen. Relativ. Gravit. 21, 125 (1989).

[16] I. Hauser and F.J. Ernst, Gen. Relativ. Gravit. 33, 1985 (2001).

[17] J.L. Friedman, J.R. Ipser, and L. Parker, Nature (London) 312, 255 (1984); Y. Eriguchi, I. Hachisu, and K. Nomoto, Mon. Not. R. Astron. Soc. 266, 179 (1994); M. Salgado, S. Bonaz- zola, E. Gourgoulhon, and P. Hanesel, Astron. Astrophys. 291, 155 (1994); G.B. Cook, S.L. Shapiro, and S.A. Teukolsky, Astrophys. J. 424, 823 (1994); N. Stergioulas and J.L. Friedman, ibid. 444, 306 (1995).

[18] T. Nozawa, N. Stergioulas, E. Gourgoulhon, and Y. Eriguchi, Astron. Astrophys., Suppl. Ser. 132, 431 (1998); J.A. Font et al. Phys. Rev. D 65, 084024 (2002).

[19] S. Bonazzola, E. Gourgoulhon, and J.-A. Marck, Phys. Rev. D 58, 104020 (1998).

[20] F.J. Chinea and L.M. González-Romero, Class. Quantum Grav. 9, 1271 (1992)

[21] L.M. González-Romero, Class. Quantum Grav. 11, 2741 (1994).

[22] S.W. Hawking and G.F.R. Ellis, The Large Scale Structure of Space-time (Cambridge University Press, Cambridge, England, 1973).

[23] R. Adler, M. Bazin, and M. Schiffer, Introduction to General Relativity (McGraw-Hill, New York, 1965).

[24] The surface $\Sigma$ is static in the sense that the induced metric admits a Killing field $k=\partial_{t}$ such that $d k_{\downarrow} \wedge k_{\downarrow}=0$ where $k_{\downarrow}$ is the 1 -form obtained from $k$ using the induced metric. 